\section{Conducting research in a resource-constrained environment: avoiding the pitfalls}

\author{
Janine I. Munsamy, ${ }^{1}$ Andy Parrish, ${ }^{1,2}$ \\ Gavin Steel ${ }^{1,3}$ \\ 'Department of Pharmacy, Rhodes \\ University, Grahamstown; ${ }^{2}$ Department \\ of Internal Medicine, Walter Sisulu \\ University and East London Hospital \\ Complex, East London; ${ }^{3}$ National \\ Department of Health, Pretoria, South \\ Africa
}

\section{Abstract}

Practical challenges affected the conducting of a retrospective drug use evaluation (DUE) on the rational use of tenofovir in a resourceconstrained South African Antiretroviral Treatment Programme. The primary outcome measure was the percentage of patient records compliant with DUE criteria using initiation prescriptions from March 2009 to February 2010. Health system challenges encountered included stringent institutional administrative procedures, lack of efficient communication channels, reliance on overburdened personnel and fear of audit. Forty percent (222 of 556) of patient records identified for inclusion in the study had to be excluded, mainly due to poor record keeping. Research budgetary constraints also limited data collection. This experience highlighted real, unforeseen challenges when conducting a retrospective study in a resource-constrained environment. A sound understanding of the environment and adequate preparation is recommended. The lessons learnt may prove valuable to both firsttime and experienced researchers in a resource-limited setting using a similar methodology.

\section{Introduction}

Review of the primary literature revealed a paucity of data evaluating the effectiveness of rational medicine use interventions in developing countries. ${ }^{1}$ During 2009-2011, a retrospective drug use evaluation (DUE) evaluated the rational use of tenofovir at accredited public sector antiretroviral treatment sites in the Province of the Eastern Cape, South Africa. DUE is a tool to improve the quality, safety and cost-effectiveness of medicine use. ${ }^{2}$ The World Bank described the economy of South Africa as upper-middle income in 2013. ${ }^{3}$ Statistics South
Africa, however, reported that the Province of the Eastern Cape has the second highest poverty levels in the country. ${ }^{4}$ There appear to be no published retrospective studies of a similar construct in the context of a resource-constrained environment. This study provides local data regarding the impact of strategies to improve rational medicine use.

A reflection on the health system challenges encountered by researchers in resource-limited settings has been reported in the context of clinical trials., ${ }^{56}$ Mbuagbaw and colleagues described their experiences regarding the administrative, ethical and financial challenges during the conducting of the Cameroon Mobile Phone SMS trial. ${ }^{5}$ The authors however acknowledged that there is a lack of data on the operational changes in low-income countries.

Research practice is enhanced by experience and lessons learnt. ${ }^{7}$ The report describes the health system challenges that impeded the research process during a retrospective study in a resource-constrained South African public sector environment.

\section{Materials and Methods}

The primary outcome measure was the percentage of patient records compliant with the DUE criteria using initiation prescriptions from March 2009 to February 2010. Data were collected in 2010 and 2011 following ethical approval by the East London Hospital Complex and Rhodes University, Faculty of Pharmacy Research Ethics Committees. Permission to access the sites was obtained from the Eastern Cape Department of Health Epidemiological Research and Surveillance Management Directorate, health care facilities and department managers.

\section{Results}

Record availability and quality. Of the 556 patient records identified for inclusion in the study, 222 (40\%) were excluded, mainly due to poor record keeping systems. Of the 222 patients records excluded, 106 (48\%) could not be found, $74(33 \%)$ were incomplete and the remaining 42 (19\%) did not meet study inclusion criteria.

Staff availability. The feasibility of data collection depended on assistance from the staff. With high patient loads and current staff shortages, this assistance was understandably not always available, especially during busier periods. During the study period there was a high turnover of pharmacy personnel with $73 \%$ (7 of 11 pharmacists) changing during the survey period. Of the 7 pharmacists, $6(88 \%)$ were community service personnel. In South Africa
Correspondence: Janine I. Munsamy, Department of Pharmacy, Rhodes University, Drosty Rd, 6139 Grahamstown, South Africa.

Tel. +2743.709 .2486 - Fax: +2746.603 .7350 .

E-mail: Janinemunsamy@yahoo.com

Key words: research, challenges, public health, resource-constrained.

Acknowledgements: the authors would like to thank the management, professional and administrative staff at the ten study sites in the Amathole District, Province of the Eastern Cape, South Africa; Prof S.E. Radloff, Department of Statistics, Rhodes University, Grahamstown, South Africa; Prof B.J. Wilson, Faculty of Pharmacy, Rhodes University, Grahamstown, South Africa.

Contributions: MJI, data collecting; MJI, PA, manuscript writing; MJI, PA, SG, manuscript review.

Conflict of interests: the authors declare no potential conflict of interests.

Funding: the work was supported by a Health Professions Training and Development (Eastern Cape Department of Health, South Africa) grant.

Received for publication: 5 February 2013.

Revision received: 1 October 2013.

Accepted for publication: 3 November 2013

This work is licensed under a Creative Commons Attribution 3.0 License (by-nc 3.0).

(C) Copyright J.I. Munsamy et al., 2014

Licensee PAGEPress, Italy

Healthcare in Low-resource Settings 2014; 2:1009 doi:10.4081/hls.2014.1009

pharmacists are required to undertake one year of remunerated pharmaceutical service in a public sector health care facility after completing their training. This interrupted data collection continuity as community service personnel identified to assist during the project plan were no longer employed at the site when data collection commenced.

Research approval delays. Institutional administrative procedures required that permission be obtained from both the health care facility and department managers following review of the research documentation and project plan. Considerable time was spent identifying and locating managers who were often unavailable (attending meetings, on leave, or other unknown reasons.) This resulted in a delay in starting data collection at certain sites as did a lack of efficient communication channels e.g. sites with only one telephone available, sites with telephone lines out of order and/or sites with no email or facsimile facilities.

Lack of staff clarity on the purpose of the research. The perception that the study formed 
part of a managerial audit process led to initial resistance to providing assistance despite facilitating documentation from Ethics Committees and the Department of Health. It can be argued that a lack of a culture of research and concerns about previous audits may have contributed to this challenge.

Budgetary constraints. The calculated sample size indicated that data should be collected from 47 sites that complied with study inclusion criteria. However research budgetary constraints meant that data could only be collected from 10 sites, limiting the statistical power of the study. Health system challenges added $75 \%$ (95\% CI; $41-93 \%)$ to the time estimated for preparation of the data collection (6 of 8 weeks of preparation time). The additional cost for employing a pharmacist for 6 weeks ( $8 \mathrm{~h}$ per day; 5 days per week) is estimated to be R58 800 (approximately R245 per h for pharmacist grade 2 , level 1$){ }^{8}$

\section{Discussion}

\section{Record availability and quality}

Generally it is expected that data from studies employing a retrospective study design would be extracted from patient records. A retrospective design study offers convenience for a study involving multiple sites, a large sample size and short data collection period however is reliant on accurate record keeping and adequate record access. Considering the inherent nature of a retrospective study coupled with poor record keeping systems in a resource-limited setting it could be argued that having adequate, good quality data may be compromised. It is recommended that standards regarding clinical records should be developed, implemented and sustained with the relevant training and ongoing audit. The introduction of electronic record systems to maintain patients' clinical data should be considered through external funding sources e.g. non-governmental organizations. Consideration should be given to concurrent or prospective research design in low-resource settings as this method of data collection is more robust.

\section{Staff availability}

Conducting a study with a limited budget imposes a burden on overworked local staff as public health facilities are burdened by extremely high patient numbers and staff shortages. In a resource-constrained environment without an established research ethos, both administrators and clinical staff may find the process challenging, the former because of the need to understand and accept research plans, and the latter because of concerns that data collection is really designed for punitive audit. All of these factors may limit the quality of the information gathered and skew the primary finding. It is recommended that Eastern Cape Department of Health implement educational interventions e.g. workshops to promote a culture of clinical research at all levels of health care. The potential for clinical research may not only be limited to health care facilities offering a specialist tertiary oriented service as the management of HIV/AIDS is a primary health care service in the South African public health sector. Staff at the sites where the study will be conducted should be well informed about the rationale for the study and potential benefit of the findings in terms of the implication for clinical practice. This information will highlight the importance of the study and may encourage participation despite resource limitations. The staff could also be invited to participate at the stage of protocol design as another educational strategy to create awareness about clinical research. Integrating the staff from this stage of the design may also promote participation. Staff involved in the study at this level should be informed that their contribution will be duly acknowledged on publication.

In 2001 South Africa implemented community service for pharmacists as part of a strategy to cope with the problem of lack of human resources in the public health sector and to improve provision of health services. However the scarcity of human resources is an ongoing struggle in the resource-constrained South African public health care sector. If community service personnel are in key positions data collection should be completed during their tenure. If this is not possible, the investigator should be aware of changes early in the study and plan accordingly. It is important to agree on the timing of data collection if the assistance of on-site staff is required.

\section{Administrative and communication challenges}

Public health facilities should develop mechanisms for granting permission for studies expeditiously once written approval has been obtained from academic bodies and provincial health departments. These health system challenges delayed study completion, with increased staff time translating into increased costs. The additional administrative burden also increased telephone costs although this was not quantified. Conducting a pilot study may have assisted in identifying some challenges earlier, allowed better planning of the data collection phase and resulted in time and cost savings.

\section{Conclusions}

This research experience highlighted unforeseen challenges when conducting a retrospective study in a resource-constrained environment. A sound understanding of the environment including the research processes and culture, anticipating and preparing for challenges and, collaboration with key personnel is recommended to circumvent unexpected pitfalls. The lessons learnt may prove valuable to both first-time and experienced researchers in a resource-limited setting using a similar methodology.

\section{References}

1. Holloway K. Combating inappropriate use of medicines. Expert Rev Clin Pharmacol 2011;4:335-48.

2. SHPA Committee of Specialty Practice in Drug Use Evaluation. SHPA standards of practice for drug use evaluation in Australian hospitals. J Res Pharm Pract 2004;34:220-3.

3. World Bank. World Bank list of econonics. World Bank ed., 2013. Available from: http://siteresources.worldbank.org/DATASTATISTICS/Resources/GDP.pdf

4. Statistics South Africa. Poverty. Statistics South Africa ed., 2012. Available from: http://beta2.statssa.gov.za/?page_id=739\& id $=1$

5. Mbuagbaw L, Thabane L, Ongolo-Zogo P, Lang T. The challenges and opportunities of conducting a clinical trial in a low resource setting: the case of the Cameroon mobile phone SMS (CAMPS) trial: an investigator initiated trial. Trials 2011;12: 145.

6. Lang TA, White NJ, Tinh Hien T, et al. Clinical research in resource-limited settings: enhancing research capacity and working together to make trials less complicated. PLoS Neglect Trop D 2010;4:e619.

7. Mirele-Cabodevila E, Stoller JK. Research during fellowship. Chest 2009;135:1395-9.

8. Department of Public Service and Administration. Cost-of-living adjustment for personnel on salary levels 1 to 12 and those covered by OSDs: 1 May 2012. Department of Public Service and Administration, Republic of South Africa ed., 2012. Available from: http:/www.dpsa. gov.za/dpsa2g/r_documents.asp\#annual_c ol_adj 